\title{
Requirements for AI-based Teammates: A Qualitative Inquiry in the Context of Creative Workshops
}

\author{
Edona Elshan \\ University of St.Gallen \\ edona.elshan@unisg.ch \\ Gert-Jan de Vreede \\ University of South Florida \\ gdevreede@,usf.edu
}

\author{
Dominik Siemon \\ LUT University \\ dominik.siemon@lut.fi
}

\author{
Sarah Oeste-Reiß \\ University of Kassel \\ oeste-reiss@uni-kassel.de
}

\author{
Triparna de Vreede \\ University of South Florida \\ tdevreede@usf.edu
}

\author{
Philipp Ebel \\ University of St.Gallen \\ philipp.ebel@unisg.ch
}

\begin{abstract}
Innovation requires organizations to tap into the knowledge and creativity of teams. However, teams are confronted with massive amounts of data and information, necessitating a broad set of knowledge, methodologies, and approaches to solve problems and innovate. Consequently, team composition has become a critical challenge. Recent advances in artificial intelligence (AI) may assist in addressing this challenge. As AI is permeating both business and private sectors, organizational teams may be augmented with AI team members. However, given the nascent nature of this phenomenon, little is known about the specific roles and requirements for such AI teammates. Within an interview study we discover common challenges in teams and identify recurring capability gaps of participants and behaviors that negatively impact the team's collective performance. Based on our findings, we propose requirements for AI-based teammates to address these gaps and support beneficial collaboration between humans and AI in teams.
\end{abstract}

\section{Introduction}

As organizations continue to face the pressure to continuously offer new products and services [1], digitization offers the potential for organizational transformation and joint value creation for complex problems (e.g., crowdsourcing, open innovation) [2]. In this complex yet enriching environment, innovations are critical to create competitive advantage and ensure sustained success [3]. Organizations need to innovate and solve problems efficiently and effectively [4]. To this end, one common approach is to work in teams that bring together different expertise, skills, and behaviors to achieve increased performance [4, 5]. Team collaboration occurs when two or more individuals work together, either in person or virtually, toward a common goal to produce outcomes for which they share responsibility. Team members depend on each other to accomplish tasks and influence outcomes through their interactions [6]. This involves going through specific phases and methods and working on certain tasks collectively, leveraging the strengths of each team member [22].

Teams often are faced with large amounts of data and information, as well as with ever shorter expiry times for information relevance [7]. Complex problems require diverse knowledge, methods, and approaches and integration of the most recent information [8]. These dynamic situations highlight the need for an effective and well-composed team to solve complex problems innovatively in a timely manner [9]. However, it often is not until the collaboration process is underway that the lack of certain roles or skills becomes apparent [9, 10]. This creates gaps and challenges that often cannot be addressed by the human team members on the fly.

One approach to address these gaps or capabilities is to use artificial intelligence (AI)-based systems that are included as partners in collaboration process and can provide the missing components and thereby closing the skills and process gaps $[11,12,13]$. AI-based systems are applications that perceive their environment, mimic human cognitive functions, to take or suggest actions towards a goal [14]. Scholars argue that humans and AIbased systems have complementary capabilities that can be combined to augment each other and achieve shared value generation $[11,13]$. A key aspect of these humanAI pairings is that tasks are performed collectively, and interdependent activities are coordinated $[15,16]$. If these joint activities are used to achieve a common goal, AI-based systems become team members in a collaboration scenario $[15,16]$.

In recent years, research on AI-based systems as part of collaboration processes has gained considerable interest. For example, Bittner et al. [13] developed a 
taxonomy of design option combinations for Conversational Agents (CAs, a specific form of AI) in collaborative work and distinguished between AI as facilitator, peer or expert in various collaboration settings [17]. In addition, Poser and Bittner [18] conducted a systematic literature review and found that CAs are mainly purposed for task-related activities and not for relationship-related activities in teamwork. As a specific example in collaborative writing, Wiethof et al. [19] investigated the UI design principles for CAs that encouraged optimal user acceptance. In doing so, they primarily considered the construct of social presence and focused on functional aspects such as natural language processing (NLP) or error-handling capabilities. Debowski et al. [20] also focused on design thinking workshops as a form of creativity workshops and derived design principles for a virtual collaborator based on expert interviews with facilitators and participants in the automotive industry. They concluded that people expect an AI to assist rather than be an equal participant in the collaboration process. They reason that this is mainly due to the common perception that AI is meant to relieve human participants of extra burden in their work. Siemon et al. [16] also propose an approach of generating team roles which determines possible tasks and also identifies the skills and behaviors of AI-based team members in collaboration scenarios. These new roles are based on existing role concepts from human-human collaboration research.

Even though these studies highlight important aspects of human-AI collaboration research, they are restricted in their application. First, these studies mostly focus on CAs, AI-based systems that interact with their users through conversation. Consequently, this represents a limited view of collaboration scenarios, as collaboration often goes beyond conversation and includes cooperation and coordination activities as well (e.g., creating a MindMap). Second, these studies follow a similar research procedure: they ask workshop participants or facilitators how AI-based systems can support them and which tasks they think that the AI should take on. This may lead to a skewed response pattern that focuses on AI-based systems as assistants to facilitators or participants. In addition, the results may be biased by the current notion of AI with limited capabilities which leads to a narrow specification of requirements. While this method was appropriate for these studies as they focus on specific use cases, it yields results that are applicable to specific and ad hoc collaborations.

However, when considering recurring collaboration challenges across multiple workshops, a broader perspective is needed to gather insights on the type of tasks that can be executed by an AI-based system to support collaboration. This forms the basis for developing reusable collaboration designs for workshops. In this study we use an exploratory approach to identify the recurring gaps in participants' skills and behaviors in creative collaboration workshops. In addition, we also explore how team capabilities can be augmented by closing these with the use of AI-based teammates.

Therefore, the goal of this paper is two-fold. First, we aim to identify recurring challenges and gaps in collaborative processes that commonly occur during workshops and are directly attributable to the individual skills of the participants. That is, collaboration process steps human participants frequently miss, either due to their limited skills or due to their constrained behaviors. Second, we derive an initial set of requirements for different roles that AI-based systems can take on as teammates, including certain skills and behaviors. In doing so, we provide answers to the research questions:

RQ1: What are common challenges in and gaps (regarding skills and behavior) of participants in collaboration processes (workshops)?

RQ2: What are the requirements for the roles that AI-based teammates can take to fill the gaps in the collaboration processes?

We followed a qualitative research approach and interviewed workshop facilitators about their experiences with specific challenges related to workshop participants, processes, and activities. Our data show recurring problems from which we derive concrete skills and behaviors that are often missing in collaboration workshops. Based on our findings, we propose a set of requirements for an AI-based team member that can play an active role in future hybrid workshops.

The remainder of this paper is structured as follows. First, we present additional background on collaboration and human-AI collaboration in teams. Next, we present our method, followed by our results. Finally, we discuss our findings, implications, and limitations of our study as well as directions for future research.

\section{Background}

\subsection{Collaboration and Teams}

Collaboration concerns joint effort towards a group goal. It includes aspects like team building, productivity, continuity, and success [21]. Collaboration also includes activities like communication, planning, coordination, adaptation, and assignment of responsibility. In addition, a traditional human-human collaboration involves individual engagement, mutual influence, and the incorporation of individual strengths and behaviors $[21,22]$. 
Collaboration typically takes place in virtual or inperson settings where all team members can contribute, and their skills, knowledge, and approaches are utilized. One of the key aspects of team collaboration is the complementarity of the team members' skills towards the team's goal achievement $[23,24]$. Team members may differ not only in terms of individual abilities, knowledge, experience, and specific skills but also in terms of socio-cognitive characteristics and behaviors [24]. Team members may also differ in their thinking patterns, abilities to develop information associations, and in their approaches to problem-solving [24, 25]. Thus, forming teams that can take advantage of these individual differences, while complex, is critical for team success $[5,9,25]$. Lack of necessary team member skills, behaviors, or thinking patterns often lead to the failure to achieve team goals [5]. Even though there may not be a 'perfect' team composition, it is important to assemble a heterogeneous team in terms of mindsets and skills such that no crucial skill that is needed to successfully complete the task is missing. Advanced AIbased systems may offer organizations a way to supplement teams with missing critical skills and capabilities.

\subsection{Human-AI Collaboration}

New technological developments are increasingly enabling IT to take on active roles in collaboration [26]. Technological capabilities of AI-based systems have elevated the complexity of interaction and collaboration between humans and AI-based systems to an unprecedented level $[13,16]$. The role of AI-based systems has evolved from being reactive and assistive technology to being a partner to humans in the collaboration process [11]. For example, AI technologies like Siri and Alexa are changing how we interact and coexist with technology [26, 27]. Such developments allow for natural interactions such that AI-based systems are no longer perceived as supportive tools but as collaborative partners that facilitate mutual value creation [13, 16, 28]. Furthermore, the perception of an AI-based system as a collaborative partner is strengthened by its increasing capabilities to express anthropomorphic characteristics like autonomy, empathy, personality, and emotions [27, 29].

The advent of CAs is a major milestone in the human-AI collaboration [14, 27]. CAs are social agents created to carry out conversations with humans $[26,30$, $31]$. Modern CAs use the power of artificial intelligence to respond to complex queries of its users' $[2,32]$. A prominent example of a CA is A.L.I.C.E. (Artificial Linguistic Internet Computer Entity), a chatbot built using the AI Mark-up Language (AIML) which utilizes natural language processing to interact with users.
CAs are regularly used in educational settings [33], in software engineering by supporting teams [34], health professional teams $[35,36]$, or as facilitators in idea generation $[37,38]$. Other times CAs are used to establish hybrid teams in the music industry [39], hospitals [36], joint value creation in the context of smart services[40], or as teammates in video games [41]. Moreover, as AI-based systems are becoming increasingly anthropomorphic $[15,28]$, they are not only being perceived as more human, but they also evoke a social presence that allows them to be integrated as partners in a collaborative process [15, 28, 42].

While AI-based systems may approximate humans in their behavior and appearance, humans and AI-based systems still possess different yet complementary capabilities that can be combined to create mutual value $[11,43]$. For AI-based systems to be effective team members rather than just supportive tools, it is important that they take on suitable and consistent tasks and thus fulfill a certain role within the team [44]. Moreover, high-functioning teams typically leverage the strengths of all team members, whether human or AI-based [24]. One approach that describes the complementary collaboration between humans and AI-based systems is hybrid intelligence. Hybrid intelligence occurs when "humans and computers have complementary capabilities that can be combined to augment each other" [12, p. 3]. The concept of hybrid intelligence suggests that optimal results are achieved when the capabilities of humans and AI-based systems are combined to create mutual value by reinforcing and complementing each other $[11,27,43]$. The main aspect of this concept is that tasks are performed jointly by humans and AI, and dependent activities are coordinated. Doing so leverages both the skills and purposeful behaviors of human team members and the capabilities of AI-based team members [11, 15], thereby enabling an effective collaboration between humans and AI-based systems. Therefore, hybrid teams must have a balanced distribution with consistent role distributions and task areas $[11,24,44]$ between humans and AIbased systems.

Hybrid intelligence is seen as a promising approach for future human-AI teams creating a happy medium between letting AI-based systems solve complex problems on their own and creating purely human teams $[11,12,36]$. When there are complex problems to be solved that require a collaborative approach, the capabilities of AI-based team members could supplement the gaps in human capabilities. This creates a richer team in terms of functionality and effectiveness. Even though the capabilities of the AI-based systems have been explored in the literature, little is known about the tasks and roles that AI-based systems should assume in human-AI collaboration processes. Yet, this 
is a crucial part of the dynamic of successful collaboration teams and is the basis for designing and deploying effective human-AI collaboration processes.

\section{Method}

To answer our research questions and identify recurring challenges and gaps in collaboration processes, we rely on a qualitative research design using expert interviews [45, 46]. Based on the experts' experience, we aim to identify recurring gaps and challenges that often occur in collaborative work scenarios, such as workshops. We asked what skills and behaviors are often missing in human participants, what difficulties this leads to, and how these could be remedied. The experts' feedback informs possible and important requirements for AI-based team members and their relevant skills and behaviors. The interviews were semi-structured, i.e., they followed a sequence of openended questions to which the interviewee could provide frank responses [46]. The interview guide supports the interviewer in ensuring that all critical aspects of the research question are addressed. Appendix 1 shows the semi-structured interview guide. During the interviews, the experts were allowed to deviate in their answers from the question sequence; interviewers would also ask follow-up questions in case the experts' responses were unclear or lacking sufficient detail.

Experts were selected as a sample of convenience from the authors' professional networks. To ensure that the experts could draw on extensive experience with creative workshops and identify recurring gaps of skills of their participants, each expert had to meet the following criteria: at least three years of experience in designing and conducting workshops, and experience with facilitating at least ten workshops. In total, we interviewed eight experts, as we felt that our data saturation was reached. The experts in our sample represented on average 7.38 years of experience and had designed and facilitated on average more than 270 workshops (combined over 2000 workshops). Table 2 provides an overview of our experts.

Table 1. Interview panel

\begin{tabular}{|l|l|l|}
\hline ID & Work experience & $\begin{array}{l}\text { Experience } \\
\text { (years) \& } \\
\text { Number of } \\
\text { Workshops }\end{array}$ \\
\hline EX1 & $\begin{array}{l}\text { Research assistant in } \\
\text { Information Systems (IS) \& } \\
\text { Design Thinking coach }\end{array}$ & $\begin{array}{l}\text { y. \& around 20 } \\
\text { workshops }\end{array}$ \\
\hline EX2 & $\begin{array}{l}\text { Design Thinking coach, agile } \\
\text { work and innovation } \\
\text { consultant }\end{array}$ & $\begin{array}{l}12 \text { y. \& around } \\
600 \text { workshops }\end{array}$ \\
\hline EX3 & Senior researcher in IS & $\begin{array}{l}6 \text { y. \& around 40 } \\
\text { workshops }\end{array}$ \\
\hline
\end{tabular}

\begin{tabular}{|l|l|l|}
\hline EX4 & $\begin{array}{l}\text { Design Thinking Coach \& } \\
\text { Consultant }\end{array}$ & $\begin{array}{l}10 \mathrm{y} . \& \text { around } \\
1000 \text { workshops }\end{array}$ \\
\hline EX5 & Research assistant in IS & $\begin{array}{l}3 \mathrm{y} . \& \text { around } 10 \\
\text { workshops }\end{array}$ \\
\hline EX6 & $\begin{array}{l}\text { Design Thinking coach \& } \\
\text { Research assistant in IS }\end{array}$ & $\begin{array}{l}3 \mathrm{y} . \text { \& around 50 } \\
-100 \text { workshops }\end{array}$ \\
\hline EX7 & $\begin{array}{l}\text { Associate professor in } \\
\text { Business Administration \& IS }\end{array}$ & $\begin{array}{l}11 \text { y. \& around } \\
500 \text { workshops }\end{array}$ \\
\hline EX8 & $\begin{array}{l}\text { Associate professor in IS \& } \\
\text { creativity expert }\end{array}$ & $\begin{array}{l}10 \text { y. \&around } \\
15 \text { workshops }\end{array}$ \\
\hline
\end{tabular}

The expert interviews took place between April and May 2021 and took, on average 40 minutes. The interviews were conducted by the authors, using the guidelines described above. Each interview was recorded, transcribed, and analyzed using the qualitative data analysis software MAxQDA (2021). Two of the authors coded the interviews independently, and the content was transferred into codes and subcodes. Using a consensus approach, differences in coding were discussed and resolved until a consensus view was reached. This resulted in an intercoder reliability of 94 $\%$.

Furthermore, the coding iterations and deriving of the requirements were iteratively validated by two researchers in research discussions [47]. This shall account for stable, valid, and reproducible research results. However, we note that when deriving requirements from interviews, there is always a certain residuum of subjective bias as individual human judgment is involved. In total, we coded six main categories (information about the experts, workshop challenges, AI-based teammate, problems of workshop activities, perfect participant, skills and behavior of participants), 74 subcodes, and assigned 472 code segments within the 8 interview transcripts.

\section{Results}

Our process of inductively coding the interviews produced a number of findings, which we subsequently grouped into five basic categories: psychological blockages, sociological blockages based on Schlick [48], as well as skill and behavior gaps, perfect participant, and AI-based teammate. Following, we summarize recurring problems and gaps for each of the categories. This constitutes the basis to derive challenges that an AI-based teammate can cope with. Psychological blockages describe things like closed mindsets, fear of change, lack of motivation, or lack of curiosity. Sociological blockages include the lack of recognition, too much competition, or other conflicts and tensions. The skill and behavior gaps category refers to overarching abilities that participants most often do not possess, while perfect participants represent the view of the experts of how a "perfect" participant should be. The AI-based teammate category picks up on this 
aspect and describes the experts' opinions regarding the skills and behaviors that an AI-based team member should possess.

Psychological Blockages. Overall, our research shows that there are numerous barriers impeding innovation during workshops. Our experts, for example, claimed that participants had the wrong mindset and would not open up. This frequently coincided with the participant's personality. As a result, participants were frequently "bored," "overwhelmed," or "too hesitant" to speak up. It was also suggested that participants should have the confidence to speak up and dispute the opinions of others. Especially when the workshop participants were students completing a case for a firm, they would not question the challenge posed by the firms. This can be seen in other application domains as well. Our expert facilitators, for example, noticed that even at internal business workshops, some participants are too hesitant and fearful of offering their ideas. However, this causes a variety of issues, which are explored in greater detail below. Some of these difficulties or hurdles arise when participants are unable to adapt to their surroundings and feel uncertain. The following quote exemplifies this: "And if they do not understand, for example, an instruction, they get annoyed very fast because they do not know how to perform. Then they do not know the frame for their performance." (Expert 6)

Sociological Blockages. The experts mentioned various sociological blockages within their workshops, which concern the difficulty of forming a group or determining which people should be put together in a group. This is heavily influenced by the individuals' personalities and the characteristics and skills they bring with them.

The following quote exemplifies how strongly group division influences other aspects of collaboration: "If you had teams where people knew each other well and had really good connections, consensus building is also something that happens easier because you know where people come from, and you know there may be stereotypes within that group." (Expert 7)

The experts further explained that coordination issues occur in addition to the problem of reaching an agreement; thus, it is preferable if participants are already acquainted with one another. This also reduces the difficulty of speaking with one another compared to when participants are unfamiliar with one another.

Experts shared various ways of group formation. While one expert (EX4) ensures that loud and quiet participants are not mixed in the same group, another expert (EX3) ensures that groups are as diverse as possible. Expert 4 states: "And I do not mix them with the noisier ones in the hope that the silent group will be able to do something as well, though I did try to mix people and personalities in general." Said Expert 3:
"[...] put individuals in a group who are not all homogeneous".

Several issues arose in terms of team dynamics. For example, it was said that as the size of the group grows, more and more people lose interest in the group and fade out. Expert 2 emphasizes this point particularly well, stating: "If the group structure is not good and the energy level is low, people go back into their old patterns $[\ldots] "$..

As a result, group dynamics have a significant influence on how attentive the individual members are. According to our interview data, the group dynamic evolves in such a way that someone in the group may assume the role of team leader at any given time. However, if more than one person wishes to fill that role, it can create extra complications. This is demonstrated by Expert 4's observations: "Everyone wants to place themselves in the best manner possible, and then you get into the storming phase where you try to battle for your position and make sure you are being heard, and then it goes into norming and performing, so this is something I can completely identify to."

Another challenging aspect is when people do not allow others to contribute. Our interviews indicated that these individual team members are frequently seen as "Alpha Persons" and have a significant impact on the sociological aspects of the workshop. These alpha individuals are distinguished by their outgoing demeanor, a big proportion of speaking time, strong ideas, and dominance during discussion. These individuals may also act so that they do not allow others to speak or even interrupt them. This is due in part to their conviction in their views and thinking. The following statement succinctly illustrates these observations: "In this case, you have an opinion leader in a group who is more extroverted, outgoing, and perhaps more involved. And this person can occasionally override all of the other people's viewpoints. [...] and says: let us make a decision, I believe we should take this one. " (Expert 1).

Skill and Behavior Gaps. However, many of the above-mentioned challenges are also due to a lack of skills, competencies, and appropriate behavior during workshops. As a result of our data collection, we were able to discover various gaps, both in terms of participant capabilities and in terms of participant behavior.

One of the most significant concerns in this regard was that many of the participants were perceived to have a knowledge gap in terms of methods. On the one hand, this concerned the fundamental understanding of the approach and its applicability. On the other hand, the range of applicable approaches left much space for improvement as well. For example, the majority of the participants had no idea how to tackle a problem. As a 
result, participants frequently feel overwhelmed and lack trust in the method and its implementation. Several times during the interviews, it was mentioned that many participants would want to have clear tasks assigned to them. While managing a team by separating the team members by role or task may be appropriate, our interviews show that this might lead to (1) not working together on the solution and (2) not building shared understanding.

While those with little awareness of workshop techniques feel overwhelmed, individuals with great understanding are frequently bored and fade out when the methodical workshop approach is discussed. According to our interviews, this is highly related to the participants' curiosity. The following quote exemplifies this: "I believe there is someone in every group who is not really interested and does not understand why they are sitting there or doing this." (Expert 3)

Another skill that participants are perceived to lack is the ability to think critically. This is especially important when an idea is to be evaluated and selected. This applies not only to their ideas but also to the ideas of other group members, provided that the other members can communicate their ideas. According to our data, challenges to critical thinking are rooted in the participants' poor understanding of their initial assumptions within a workshop (e.g., customer needs). Thus, it would be beneficial if participants deliberate deeply and really try to get deep into a problem and understand it and its aspects.

An idea must first be expressed in order to be assessed and then selected. According to our experts, this is one of the most significant gaps in participants' skills. There are various gradations here. Some people cannot or do not want to formulate their views because they are too bashful or do not want to be included in the process. Others blend a variety of concepts and are unable to establish a clear concept. Some experts emphasized that many participants also like to formulate their opinions in a broad fashion rather than giving a precise description.

The foregoing deficiencies in skills and abilities are detrimental to teamwork. This results, according to our experts, mostly in the absence of creativity. This issue may grow as a result of the other deficits. For example, a group may be unable to formulate its thoughts and must therefore rely on tried-and-true formulas, which can be categorized as "not-outside-the-box" thinking. This may be due to the participants' shyness. People who are too shy are unlikely to take chances in forming and sharing ideas, and so it is less likely that innovative or novel solutions to the problem will emerge. It was also mentioned that many participants say what they think the facilitator wants to hear and hence do not participate in the actual creative process. Furthermore, there are groups that know exactly what their outcome and solution will be from the start. As a result, they do not engage in creative thinking as they do not perceive any need for it.

Perfect Participant. When we asked the experts what abilities a perfect participant should have and how (s)he should behave, the majority focused on the aspects they are now struggling with. For example, experts prefer prepared, open, and empathic participants. Also, participants must be able to lead a group and respond to the requirements of the other group members. Furthermore, the ideal participant must have a wide awareness of the application environment, current trends, and the method employed. In this regard, their way of thinking is equally crucial. The desired curiosity, which is an integral aspect of the mindset, stands out even more here.

When we examine what it means to lead a group and what the ideal participant should be able to accomplish or do in these group leadership activities, we can see that this skill includes various elements.

Essentially, the ideal participant should be able to operate well in a group. Following that, it would be advantageous if the ideal participant in this function could organize the team and provide tasks to the various members to be worked on. Furthermore, the ideal participant should be able to set their personal needs aside and focus on the group's well-being, even if this contradicts their own beliefs and needs. However, this also implies that (s)he should be able to step back and serve the group. Furthermore, the ideal participant should be able to hold others accountable, while praise should be used to strike a balance. As a result, the ideal participant should express gratitude and highlight someone successfully solving a problem.

When we examined what it means to be constructive and provide feedback, we noticed that it should be delivered both inside the group and the facilitator. For example, the experts would like to hear from the group leader to determine better how they should assist the group. A great participant should always provide comments in a polite and pleasant manner. However, it should not be forgotten that critical questions should be raised instead of just sugar-coating issues. As a result, the ideal participant should also be able to say 'no' if a notion is useless or the group is moving too steadfastly in one direction. This is illustrated by the quote of Expert 2: "[...]able to say 'no' to others and to support also ideas that are not their own kind of a just and be fair in evaluating ideas. "

In addition to these abilities, it is critical that the ideal participant meticulously documents everything so that it may be understood why an idea was pursued and why it was not. Furthermore, the ideal participant should constantly work goal-oriented and keep an eye on the 
time, attempting to timebox the group if things go on for too long.

AI-based Teammate. Our findings demonstrate that the interviewed experts believe that many of the above-mentioned issues and participant deficits may be at least minimized, if not totally overcome, by the deployment of an AI-based member. In essence, an AIpowered team member should have the precise skills, talents, and behaviors of the ideal participant. This nonhuman member further provides additional opportunities and advantages beyond the capabilities of a human member. One of the most significant advantages of the AI-based team member is, in fact, its non-human nature: As a result, the AI-based team member may take on roles that have a negative connotation and may cause interhuman conflict. Consider, for example, time boxing. When it comes to interpersonal connections, an AI-based member should be able to read the energy level in the room and alter their conduct accordingly. The following quotation exemplifies this: "[...] detect participants' social cues, emotive states, and then respond to them on how they might modify that, you know, give them a little bit of a picture of where they are right now." (Expert 8)

In this way, the AI-based member should also understand the many participants and adapt its behavior to their needs and interests. It should, for example, recognize when a participant is more sarcastic than others and determine whether this is related to the context or to personality. Furthermore, the AI-based teammate should be emphatic in their treatment of all other members, and it should be unbiased and unaffected by internal politics or hierarchy.

The AI-based participant should also be able to conduct more hands-on tasks, such as delivering a summary of the session and ensuring that everything has been considered. Nevertheless, the AI-based participant should not merely be there to assist the human participants during the workshop. Instead, it should function like a coworker. This attitude is exemplified by the following quote: "[...]an engaged coworker who is eager to get things done. [....] In the end, I believe that AI should not be used solely to assist actual humans in completing challenges or tasks, rather than relieving them of labor-intensive tasks." (Expert 1)

The following table summarizes the most significant criteria, i.e., high-level requirements for the abilities or behavior of AI-based team members, along with an explanation and the problem to be solved.
Table 2. Requirements for an AI-based Teammate

\begin{tabular}{|c|c|c|}
\hline & Description & Challenge \\
\hline \multicolumn{3}{|c|}{ Activity-Focused Requirements } \\
\hline Prepare & $\begin{array}{l}\text { AI-based teammate should do } \\
\text { some research about relevant } \\
\text { topics. }\end{array}$ & $\begin{array}{l}\text { Lack of broad } \\
\text { understanding }\end{array}$ \\
\hline $\begin{array}{l}\text { Keep } \\
\text { Track }\end{array}$ & $\begin{array}{l}\text { AI-based teammate should } \\
\text { remind other teammates of tasks } \\
\text { to be completed and check the } \\
\text { progress. }\end{array}$ & $\begin{array}{l}\text { Lack of goal- } \\
\text { orientation; } \\
\text { Bad } \\
\text { Timeboxing }\end{array}$ \\
\hline $\begin{array}{l}\text { Capture } \\
\text { Team } \\
\text { Dynamic }\end{array}$ & $\begin{array}{l}\text { AI-based teammate should } \\
\text { capture the team dynamics (e.g., } \\
\text { by the tonality) in order to } \\
\text { prevent conflicts. }\end{array}$ & $\begin{array}{l}\text { Bad team } \\
\text { dynamics }\end{array}$ \\
\hline $\begin{array}{l}\text { See bigger } \\
\text { Picture }\end{array}$ & $\begin{array}{l}\text { AI-based teammate should be } \\
\text { able to synthesize different ideas } \\
\text { and see the bigger picture to } \\
\text { combine them. }\end{array}$ & $\begin{array}{l}\text { Lack of goal- } \\
\text { orientation }\end{array}$ \\
\hline Select Idea & $\begin{array}{l}\text { AI-based teammate should select } \\
\text { ideas in an objective manner. }\end{array}$ & $\begin{array}{l}\text { Lack of critical } \\
\text { thinking }\end{array}$ \\
\hline \multicolumn{3}{|c|}{ Personality-Focused Requirements } \\
\hline $\begin{array}{l}\text { Open\& } \\
\text { reflective }\end{array}$ & $\begin{array}{l}\text { AI-based teammate should be } \\
\text { open-minded and reflective. }\end{array}$ & Wrong mindset \\
\hline Neutral & $\begin{array}{l}\text { AI-based teammate should be } \\
\text { neutral and not be on the side of } \\
\text { any other teammate. }\end{array}$ & $\begin{array}{l}\text { Bad team } \\
\text { dynamics }\end{array}$ \\
\hline
\end{tabular}

\section{Discussion and Conclusion}

Research on the use of $\mathrm{AI}$ in teams is currently rather scattered $[15,49]$. Most studies focus on a single AI functionality (e.g., AI as a facilitator in workshops [38], AI to evaluate ideas [50]) rather than taking a problem-driven approach, where specific gaps are identified by human participants and can be taken over by an AI-based teammate, in line with the hybrid intelligence approach where humans and AI complement each other [49]. To the best of our knowledge, our work represents the first high level overview of specific requirements for AI-based teammates. Taking a holistic approach to see which areas could use support, the experts highlighted a wide variety of ways in which an AI-based teammate can augment teamwork in creative workshops.

Our findings also lay a foundation to extract specific roles for AI-based teammates. Current research already highlights that AI roles, in particular, should be defined in various collaboration scenarios in order to assemble functioning teams so that fruitful group dynamics can emerge $[13,17,44]$. The detailed functional requirements that have been identified can be grouped into distinct bundles that represent a specific role, for example, the AI-based teammate as a group dynamics coach or as a workshop methods trainer. Team roles entail a range of different skills and behaviors and different expectations that other team members abide 
by. This is especially important for AI-based teammates, as, for example, expecting too much from a possible omnipotent AI teammate could have a negative impact on human team members. AI-based team members should therefore not be allowed to take over all tasks and responsibilities using their intelligent, powerful, and always-available resources [11] as this could lead human team members to downscale their efforts as they would rely on the all-encompassing capabilities of the AI-based team member [51].

Finally, the high-level requirements represent a starting point for dedicated and programmatic studies to investigate the effects of specific AI-based team support on team performance in creative workshops. Experimental studies, for example, could investigate how human team member react and behave when an AIbased teammate for instance selects a specific idea. Furthermore, future research could shed light on whether the AI taking on negatively connotated tasks such as timeboxing, influences the team as well as the collaboration.

Our findings also contribute to practice. Developers can use the structure of requirements as an inspiration to develop AI-support for teams or to benchmark the functionality of existing AI-applications in this area. Furthermore, our overview of AI-teammate requirements can form the foundation of a diagnosis checklist to determine the specific needs of creative teams that regularly work together. This would enable the team leader to identify which AI-functionality might be most beneficial to be added to the team.

A number of limitations have to be considered in the context of our study. Each of these provides interesting avenues for future research as well. First, we interviewed a limited number of experts. While we experienced saturation in the qualitative data that we collected, future efforts could focus on expanding the set of experts, for example, by broadening the range of industries and cultures that they are active in. Second, our initial data collection focused on the perspectives and insights from expert facilitators. It would be useful to augment our data with insights from regular team members as well. Team members will provide a different perspective on the different interview topics. They can also reflect on the challenges they experience with team leaders or facilitators. Therefore, future research should considerate the perspective of the workshop participants and compare the findings with ours, to see where discrepancies exist. Third, people react differently to AI and its potential. Some see AI as a threat to autonomous work and decision-making, while others see AI as a great opportunity to boost performance [52, 53, 54]. We did not actively investigate the perception of the interviewed experts on $\mathrm{AI}$ and its potential to do good as well as harm. Future efforts could investigate this aspect and determine if it has any bearing on the type of support that experts may envision. Finally, our research is based on the premise that heterogeneity in terms of team competencies is desirable for the context of creative workshops. Future research is required to determine whether (1) AI applications can indeed effectively enhance such heterogeneity and (2) whether this will lead to enhance downstream effects, such as team creativity, problemsolving performance, or decision quality.

Our other plans for future research include the following: We will develop a collection of specific use cases modeled with the collaboration process modeling technique FPM2.0 [55], to depict specific scenarios for teams working with an AI teammate that has certain functionality. These use cases can then inform the development of prototypes of AI team members that realize some of the requirements, e.g., capturing team dynamics or selecting ideas. We further aim to gather process and outcome data from a large collection of electronic creative meetings that can be used as 'training data' for the AI prototypes. Finally, we plan to design experimental studies to investigate whether the AI team member prototypes will be associated with relevant team performance variables, such as level of creativity, speed of task completion, satisfaction with process and outcomes, and commitment to team outcomes.

\section{References}

[1] Anning-Dorson, T., "Innovation and competitive advantage creation", International Marketing Review, 2018. [2] Wessel, L., A. Baiyere, R. Ologeanu-Taddei, J. Cha, and T. Blegind-Jensen, "Unpacking the difference between digital transformation and IT-enabled organizational transformation", Journal of the Association for Information Systems 22(1), 2021.

[3] Azoulay, P., and B. Jones, "Beat COVID-19 through innovation", Science 368(6491), 2020, pp. 553-553. [4] Amabile, T.M., and M.G. Pratt, "The dynamic componential model of creativity and innovation in organizations: Making progress, making meaning", Research in Organizational Behavior 36, 2016, pp. 157-183.

[5] Belbin, R.M., Management Teams: Why they succeed or fail, Routledge, 2010.

[6] Mohrman, S.A., S.G. Cohen, and A.M. Morhman Jr, Designing team-based organizations: New forms for knowledge work., Jossey-Bass, 1995.

[7] Lesher, M., D. Gierten, and A. Attrey, Going Digital Integrated Policy Framework, OECD publishing, 2020.

[8] Wolf, K.M., and H.A. Mieg, "Cognitive determinants of the success of inventors: Complex problem solving and deliberate use of divergent and convergent thinking", European Journal of Cognitive Psychology 22(3), 2010, pp. 443-462.

[9] Higgs, M., U. Plewnia, and J. Ploch, "Influence of team composition and task complexity on team performance", Team Performance Management: An International Journal, 
2005.

[10] Jackson, S.E., "Team composition in organizational settings: Issues in managing an increasingly diverse work force", In Group process and productivity. Sage Publications, Inc, Thousand Oaks, CA, US, 1991, 138-173.

[11] Dellermann, D., P. Ebel, M. Söllner, and J.M. Leimeister, "Hybrid intelligence", Business \& Information Systems Engineering, 2019, pp. 1-7.

[12] Mirbabaie, M., S. Stieglitz, F. Brünker, L. Hofeditz, B. Ross, and N.R.J. Frick, "Understanding Collaboration with Virtual Assistants - The Role of Social Identity and the Extended Self", Business \& Information Systems Engineering 63(1), 2021, pp. 21-37.

[13] Seeber, I., E. Bittner, R.O. Briggs, et al., "Machines as teammates: A research agenda on AI in team collaboration", Information \& Management, 2019, pp. 103174.

[14] Russell, S.J., and P. Norvig, Artificial intelligence: a modern approach, Malaysia; Pearson Education Limited, 2016.

[15] Seeber, I., L. Waizenegger, S. Seidel, S. Morana, I. Benbasat, and P.B. Lowry, "Collaborating with technologybased autonomous agents", Internet Research, 2020.

[16] Siemon, D., and T. Strohmann, "Human-AI Collaboration: Introducing the Virtual Collaborator", Collaborative Convergence and Virtual Teamwork for Organizational Transformation, 2020, 105-119. www.igiglobal.com/chapter/human-ai-collaboration/265472 [17] Bittner, E.A.C., S. Oeste-Reiß, and J.M. Leimeister, "Where is the Bot in our Team? Toward a Taxonomy of Design Option Combinations for Conversational Agents in Collaborative Work", (2019).

[18] Poser, M., and E.A. Bittner, "Hybrid Teamwork: Consideration of Teamwork Concepts to Reach Naturalistic Interaction between Humans and Conversational Agents", 2020.

[19] Wiethof, C., N. Tavanapour, and E. Bittner, "Designing and evaluating a collaborative writing process with gamification elements: Toward a framework for gamifying collaboration processes", AIS Transactions on HumanComputer Interaction 13(1), 2021, pp. 38-61.

[20] Debowski, N., D. Siemon, and E. Bittner, "Problem Areas in Creativity Workshops and Resulting Design Principles for a Virtual Collaborator", 2021.

[21] Randrup, N., D. Druckenmiller, and R.O. Briggs, "Toward a Philosophy of Collaboration", International Journal of e-Collaboration (IJeC), 2018.

[22] Siemon, D., F. Becker, L. Eckardt, and S. RobraBissantz, "One for all and all for one - towards a framework for collaboration support systems", Education and Information Technologies, 2019, pp. 1-25.

[23] Belbin, R.M., Team roles at work, Routledge, 2012.

[24] van de Water, H., K. Ahaus, and R. Rozier, "Team roles, team balance and performance", Journal of Management Development 27(5), 2008, pp. 499-512.

[25] Saldaña-Ramos, J., A. Sanz-Esteban, J. García, and A. Amescua, "Skills and abilities for working in a global software development team: a competence model", Journal of Software: Evolution and Process 26(3), 2014, pp. 329338.

[26] Anderson, J., L. Rainie, and A. Luchsinger, "Artificial intelligence and the future of humans", Pew Research Center,
2018.

[27] Diederich, S., A. Brendel, S. Morana, and L. Kolbe, "On the Design of and Interaction with Conversational Agents: An Organizing and Assessing Review of Human-Computer Interaction Research", Journal of the Association for Information Systems, 2022.

[28] Nass, C., J. Steuer, and E.R. Tauber, "Computers are social actors", Proceedings of the SIGCHI conference on Human factors in computing systems, ACM (1994), 72-78. [29] Ahmad, R., D. Siemon, and S. Robra-Bissantz, "Communicating with Machines: Conversational Agents with Personality and the Role of Extraversion", Proceedings of the 54th Hawaii International Conference on System Sciences, (2021), 4043.

[30] Dale, R., "The return of the chatbots", Natural Language Engineering 22(5), 2016, pp. 811-817.

[31] Westerman, D., A.C. Cross, and P.G. Lindmark, "I believe in a thing called bot: Perceptions of the humanness of 'chatbots'", Communication Studies 70(3), 2019, pp. 295312.

[32] Levesque, H.J., Common sense, the Turing test, and the quest for real AI, Mit Press, 2017.

[33] Elshan, E., and P. Ebel, "Let's Team Up: Designing Conversational Agents as Teammates", 2020.

[34] Lebeuf, C., M.-A. Storey, and A. Zagalsky, "How Software Developers Mitigate Collaboration Friction with Chatbots", arXiv:1702.07011 [cs], 2017.

[35] Kowatsch, T., M. Nißen, C.-H.I. Shih, et al., "Textbased healthcare chatbots supporting patient and health professional teams: Preliminary results of a randomized controlled trial on childhood obesity", 2017.

[36] Mirbabaie, M., S. Stieglitz, and N.R.J. Frick, "Hybrid intelligence in hospitals: towards a research agenda for collaboration", Electronic Markets, 2021.

[37] Hwang, A.H.-C., and A.S. Won, "IdeaBot: Investigating Social Facilitation in Human-Machine Team Creativity", Proceedings of the 2021 CHI Conference on Human Factors in Computing Systems, Association for Computing Machinery (2021), 1-16.

[38] Strohmann, T., S. Fischer, D. Siemon, et al., "Virtual Moderation Assistance: Creating Design Guidelines for Virtual Assistants Supporting Creative Workshops", Proceedings of the 22nd Pacific Asia Conference on Information Systems, (2018).

[39] Elshan, E., C. Engel, and P. Ebel, "Opening the Black Box of Music Royalties with the Help of Hybrid Intelligence", (2021), 5525.

[40] Knote, R., A. Janson, M. Söllner, and J.M. Leimeister, "Value Co-Creation in Smart Services: A Functional Affordances Perspective on Smart Personal Assistants", Journal of the Association for Information Systems 22(2), 2021.

[41] Zhang, R., N.J. McNeese, G. Freeman, and G. Musick, " 'An Ideal Human': Expectations of AI Teammates in HumanAI Teaming", Proceedings of the ACM on HCI 4(CSCW3), 2021, pp. 246:1-246:25.

[42] Qiu, L., and I. Benbasat, "Evaluating Anthropomorphic Product Recommendation Agents: A Social Relationship Perspective to Designing Information Systems", Journal of Management Information Systems 25(4), 2009, pp. 145-182. [43] Gerber, A., P. Derckx, D.A. Döppner, and D. Schoder, 
"Conceptualization of the Human-Machine Symbiosis - A Literature Review", (2020).

[44] Siemon, D., R. Li, and S. Robra-Bissantz, "Towards a Model of Team Roles in Human-Machine Collaboration",

ICIS 2020 Proceedings, 2020.

[45] Babbie, E., The practice of social research, Nelson Education, 2015.

[46] Mayring, P., "Qualitative content analysis: theoretical foundation, basic procedures and software solution", 2014.

[47] Forman, J., and L. Damschroder, "Qualitative Content Analysis", In L. Jacoby and L.A. Siminoff, eds., Empirical Methods for Bioethics: A Primer (Advances in Bioethics,

Volume 11). Emerald, 2007, 39-62.

[48] Schlick, G.H., Innovationen von A - Z: Begriffe, Definitionen, Erläuterungen und Beispiele ; mit 13

Checklisten und 650 Literaturstellen, 1995.

[49] Daugherty, P.R., and H.J. Wilson, Human+ machine: Reimagining work in the age of AI, Harvard Business Press, 2018.

[50] Maher, M.L., and D.H. Fisher, "Using AI to evaluate creative designs", DS 73-1 Proceedings of the 2nd International Conference on Design Creativity Volume 1, (2012).

[51] Siemon, D., and F. Wank, "Collaboration With AIBased Teammates - Evaluation of the Social Loafing Effect", (2021).

[52] Dowd, M., "Elon Musk's Billion-Dollar Crusade to Stop the A.I. Apocalypse", Vanity Fair, 2017.

[53] Kane, G.C., A.G. Young, A. Majchrzak, and S. Ransbotham, "Avoiding an Oppressive Future of Machine Learning: A Design Theory for Emancipatory Assistants", [54] Ransbotham, S., D. Kiron, P. Gerbert, and M. Reeves, "Reshaping business with artificial intelligence: Closing the gap between ambition and action", MIT Sloan Management Review 59(1), 2017.

[55] Winkler, R., R.O. Briggs, G.-J. De Vreede, J.M. Leimeister, S. Oeste-Reiss, and M. Sollner, "Modeling Support for Mass Collaboration in Open Innovation Initiatives-The Facilitation Process Model 2.0", IEEE Transactions on Engineering Management, 2020, pp. 1-15.

\section{Appendix - Interview guide}

Personal questions

1. How long are you working as a workshop facilitator?

2. How many workshops have you designed or conducted?

3. In what domain do you usually conduct workshops?

4. Who are your participants typically?

5. Do you have experience with AI-based systems, such as conversational agents or virtual assistants?

General workshop questions

6. What are the biggest challenges that occur during your workshops?

7. What workshop activities are you often not satisfied with? Please explain further or give an example

8. What workshop outcomes are you often not satisfied with? Please explain further or give an example
Please take the perspective of how your workshops are perceived by the participants.

9. Think of some skills like critical thinking.

a. What gap of skills do you most often see in your workshops amongst participants?

b. What skills should a "perfect" participant have?

10. Think of some behavior like social loafing, encouraging or even disturbing.

a. What gap of behavior do you most often see in your workshops amongst participants?

b. What behavior should a "perfect" participant have?

Specific questions regarding collaboration activities

11. Imagine in your workshop participants are supposed to generate ideas and give free rein to their thoughts. The task is to come up with as many new and crazy ideas as possible, as in brainstorming, for example.

a. What challenges do you usually see during these activities?

b. How often do you experience challenges or problems during these activities?

12. Now you're at the point where your participants need to sort through the many new ideas, identify similarities, combine ideas, and reduce the sheer number of ideas.

a. What challenges do you usually see during these activities?

b. How often do you experience challenges or problems during these activities?

13. During your workshop, the participants have to coordinate themselves from time to time, clarify relevant aspects and create a common understanding and build consensus.

a. What challenges do you usually see during these activities?

b. How often do you experience challenges or problems during these activities?

14. Also, during your workshops, decisions often have to be made, a certain further offense has to be agreed upon, and ideas have to be evaluated and agreed upon.

a. What challenges do you usually see during these activities?

b. How often do you experience challenges or problems during these activities?

Team roles and archetypes

15. Maybe you have noticed that some of your participants exhibit behaviors and are endowed with certain skills that others may not.

a. Is there a recurring behavior among your workshop participants that you can observe?

b. Are there any recurring skills in your workshop participants that you can observe?

Questions for AI-based teammates

16. Now try taking these different behaviors and skills and apply them to an AI-based teammate that works as an equal partner in your workshop.

a. What skills should your AI-based teammate have? b. What behaviors should it exhibit? 